\title{
An Analogue Computer for the Solution of the Radio Refractive-Index Equation
}

\author{
Walter E. Johnson
}

\begin{abstract}
The solution to the radio refractive-index equation provides information necessary to the research worker studying tropospheric radio propagation in the very high and ultrahigh regions. Present means of computation are either time consuming or of low accuracy. An analogue computer is developed to solve this equation by utilizing basic computation circuits incorporated into a null-balance bridge circuit. A unique feature of this computer is the modification of a linear 10-turn potentiometer so that its output to input voltage ratio versus rotation closely approximates the exponential-type curve of the saturated water-vapor term in the refractive-index equation.

This computer is now in use at the Central Radio Propagation Laboratory, of the National Bureau of Standards, Boulder, Colo., and has superseded ọther methods of calculating the radio refractive index.
\end{abstract}

\section{Introductory Concepts}

Analogue and digital computation devices are not only lightening the load of mathematicians and design engineers, but also are in many cases enabling the research worker to explore problems that have, in the past, been neglected because of the volume of calculations required prior to embarking on the analysis of the actual problem. Such a case exists in radio-propagation studies in the form of atmospheric refractive-index computations.

\subsection{Development of the Basic Refractive-Index Equation}

The two parts of the equation for the radio refractive index as developed by Debye $[1]^{1}$ are: (a) a term proportional to density resulting from the induced polarization of the gas molecules in an external field; and (b) a term proportional to the permanent dipole moment of the molecules.

The latest microwave determinations of the constants of this equation yield the following expression [2]:

$$
N=(n-1) 10^{6}=\frac{77.6}{T}\left(p+\frac{4810 e}{T}\right),
$$

where

$N=$ refractivity of air,

$n=$ refractive index of air,

$T=$ absolute temperature in degrees Kelvin,

$p=$ total pressure in millibars,

$e=$ partial pressure of water vapor in millibars.

\subsection{The Refractive Index in Radio-Wave-Propaga- tion Studies}

For low angles of elevation, the curvature of a radio ray is, to a high degree of approximation, equal to the gradient or change of refractive index with height. Consequently, the radio horizon fof $\mathrm{VHF}-\mathrm{UHF}$ communications is determined solely by the refractive-index gradient. With an increase in

\footnotetext{
1 Figures in brackets indicate the literature references at the end of this paper.
}

the refractive-index gradient, the distance to the radio horizon frequently can increase fourfold and under extreme conditions may become theoretically infinite. Therefore, a knowledge of the refractiveindex profile with height would suffice for refraction studies. If, however, one is concerned with reflection from elevated layers, then one needs the additional knowledge of the distribution of refractive index throughout the layer.

In radio surveying and navigation studies the absolute value of the refractive index is needed because of the fact that the velocity of electromagnetic waves in air is inversely proportional to the refractive index.

For a typical propagation study, at least two Weather Bureau radiosonde ${ }^{2}$ stations are selected, one on each side of the radio-transmission path. Each radiosonde records data at between 15 and 20 different elevations. For 1 year's radio data, there would be at least 21,600 calculations of $N$ to be made for the two radiosonde stations, taking observations twice each day. Therefore, it can be seen that a great deal of time is consumed in laborious calculations prior to making a radio-propagation study.

\subsection{Present Methods of Calculating the Refractive Index}

One of the most common means of reducing the calculating time is the utilization of nomograms. A recent example of the nomogram approach is that of Swingle [3], which utilizes a separate nomogram for each term in the refractive-index equation and has a maximum possible accuracy of $\pm 1 N$ unit. The solutions that utilize a single nomogram are never more precise than Swingle's method, and it is not uncommon to encounter errors of $\pm 5 N$ units.

Another approach to solving the equation is to use specially constructed slide rules. Weintraub [4] utilizes 2 slide rules, 1 for each term in the refractiveindex equation, and attains an accuracy of $\pm \frac{1}{2} N$ unit.

2 The radiosonde is a measuring system designed to measure the temperature, pressure, and relative humidity of the atmosphere at elevations from sea ture, pressure, and relative hum
level to $100,000 \mathrm{ft}$ above sea level. 
A major factor to be considered is that both the nomogram and the slide-rule methods require the strict attention of the calculator, and, as such, fatigue and the human element restrict not only the average accuracy of the results, but also the number of consecutive computations possible at one time. The time necessary to compute the separate terms in the refractive index and the subsequent addition varies from approximately $30 \mathrm{sec}$, using slide rules, to from 30 to 45 sec, using nomograms.

The material to follow describes a relatively simple electric analogue computer that has been designed to solve this equation.

\section{Computer Requirements}

The general requirements that a computer designed to solve this equation must meet are much the same as those that would be placed on any computer. An ideal approach to the problem would be to make the computer as accurate, as fast, and as convenient as possible. However, it would be well to take a more realistic approach toward finding practical limits to each of these three requirements.

\subsection{Accuracy}

In general, the computer accuracy need be no greater than is consistent with the accuracy of the available data to be used. Striving for greater accuracy would only lead to greater expense and perhaps undue complications in the circuits. However, the possibility that the accuracy of the available data may at some future date be increased must be taken into account.

At present, the greater part of the available meteorological data has been obtained from radiosonde ascents. The accuracies associated with these data would dictate a computer accuracy of no greater than 1 or $2 N$ units of refractivity. However, future plans indicate that some of the data will be obtained from relatively accurate recording instruments placed at discrete elevations on specially located steel towers. To accurately solve the refractive-index equation, using data obtained in this manner, it will be necessary to specify an accuracy of $\pm 1 / 2 N$ unit.

\subsection{Speed}

A very obvious limitation on the overall time required to complete one computation is that the total time can be no less than the time required to supply information to the computer, which is proportional to the variables in the equation. This is commonly referred to as the "setup" time. In operation, the operator will be required to read data sheets, which have temperature, pressure, and relative humidity recorded as numerical values and introduce this information into the computer. Using either of the two more common media of dials or pushbuttons, it would appear well to allow at least $3 \mathrm{sec}$ for each of these operations. Allowing another 3 sec to complete the solution would indicate a figure of approximately 12 sec to obtain one solution with the computer. This figure, if met by the computer, would be a considerable improvement over the speed obtained by either the nomogram or the slide rules.

\subsection{Convenience}

In designing a computer of this type, which must perform a large number of similar computations, too much stress cannot be laid on its convenience of operation. A machine that has been designed with this in mind not only speeds up the operational time for the operator, but also greatly reduces operational errors. Certain general features emerge from a careful consideration of the convenience requirement.

The dials or pushbuttons used to introduce the variables should be arranged in the same relative order as the data on the data sheets. Also they should be convenient to read and should be calibrated in the same units as the original data. The method of reading the answer should be such that the operator may read and record the result from the same convenient position as used to set up the computer.

With these general requirements in mind, let us next investigate the basic electric circuits that will be required in an electric analogue computer designed to solve the refractive-index equation.

\section{Basic Computation Circuits}

If the constants 77.6 and 4810 in eq. (1) are replaced by $K_{1}$ and $K_{2}$, respectively, and $e$ by its equivalent, $e_{s} R H$, the saturated vapor pressure times the relative humidity, the equation will now appear as

$$
N=\frac{K_{1}}{T}\left(p+K_{2} \frac{e_{s}}{T} R H\right)
$$

It is to be noted that for the present $e_{s} / T$ will be considered as a variable, as are $T, p$, and $R H$. The discussion to follow describes the method used to eliminate $e_{s} / T$ as an independent variable and thus reduce to three the number of variables that must be introduced into the computer.

Inspection of the equation reveals that multiplication must be accomplished in obtaining the product $K_{2}\left(e_{s} / T\right) R H$. Addition is next used to add $p$ to this product, and finally, division is utilized to divide this binomial by $T / K_{1}$. The circuits to follow indicate how each of these three mathematical operations may be duplicated electrically. In each of these circuits $e_{i}$ represents a d-c voltage source, with zero internal impedance, and $e_{0}$ is the output voltage, as meastured with an infinite impedance voltmeter. The variable-resistance symbols represent linear potentiometers so designed that the voltage as measured at the slider is equal to the percentage rotation times the voltage applied to the potentiometer. $X$ is used to represent the percentage of total rotation as measured in the direction of the small arrow associated with $X$. 


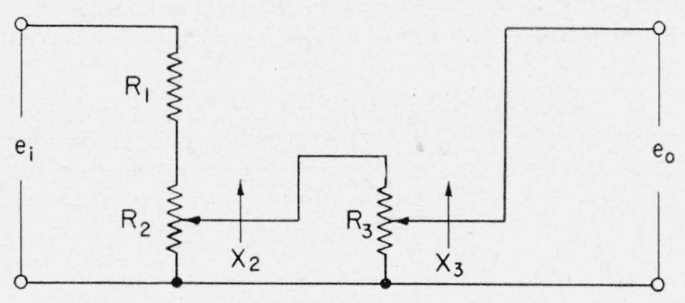

Figure 1. Multiplication circuit.

\subsection{Multiplication}

The circuit shown in figure 1 is presented as a method of multiplying two variables by a constant. The equation that expresses the ratio of output voltage to input voltage in this circuit is

$$
\frac{e_{0}}{e_{i}}=\frac{\frac{X_{2} R_{2} R_{3}}{X_{2} R_{2}+R_{3}}}{R_{1}+\left(1-X_{2}\right) R_{2}+\frac{X_{2} R_{2} R_{3}}{X_{2} R_{2}+R_{3}}} X_{3} .
$$

If $R_{3} \gg R_{2}$, the equation ${ }^{3}$ may be simplified as follows:

$$
\frac{e_{0}}{e_{i}}=\frac{R_{2}}{R_{1}+R_{2}} X_{2} X_{3}
$$

This equation shows that when $R_{2} /\left(R_{1}+R_{2}\right)$ is made equal to the desired constant and $X_{2}$ and $X_{3}$ equal to the variables, the voltage ratio, $e_{0} / e_{i}$, will equal the product of the constant and the two variables.

\subsection{Addition}

The method of adding a constant, a variable, and a constant times a variable is shown below. $R_{4 \mathrm{a}}$ and $R_{4 \mathrm{~b}}$ are identical potentiometers mounted on the same shaft. Their sliders are so phased that they both are on zero at the same time. $X_{4}$ may then be used to represent the percentage of rotation of either potentiometer. The subscripts chosen for the resistance values will prove to be convenient later when combining the basic circuits into the combined computation circuit.

The ratio of output to input voltage is given by

where

$$
\frac{e_{0}}{e_{i}}=\frac{R_{p} R_{2}}{R_{s}\left(R_{1}+R_{2}\right)} X_{2}+\frac{R_{6 \mathrm{a}}+X_{4} R_{4 \mathrm{a}}}{R_{s}},
$$

$$
R_{p}=\frac{\left(R_{1}+R_{2}\right)\left(R_{4 \mathrm{a}}+R_{8}^{\prime}\right)}{R_{1}+R_{2}+R_{4 \mathrm{a}}+R_{8}^{\prime}} \quad \text { and } \quad R_{s}=R_{p}+R_{4 \mathrm{a}}+R_{6 \mathrm{a}}
$$

3 When $R_{3}$ is 200 times $R_{2}$, the solution obtained by eq (3) shows a maximum error in $\varepsilon_{0} / \epsilon_{i}$ of 0.07 percent. This maximum error occurs at a value of $X_{2}$ equal to 66.7 percent.

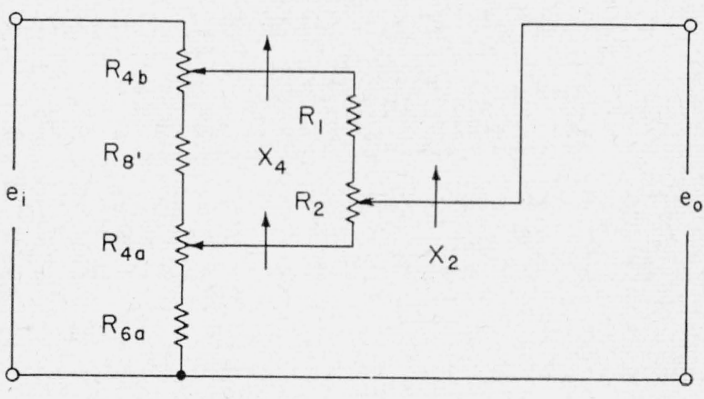

Figure 2. Addition circuit.

or, rewriting,

$$
\frac{e_{0}}{e_{i}}=\frac{R_{4 \mathrm{a}}}{R_{s}}\left[\frac{R_{6 \mathrm{a}}}{R_{4 \mathrm{a}}}+X_{4}+\frac{R_{p} R_{2}}{R_{4 \mathrm{a}}\left(R_{1}+R_{2}\right)} X_{2}\right] .
$$

If the correct values are chosen for the resistances, this circuit (fig. 2), as shown by eq (4), can be used to solve the addition of a constant, a variable, and a constant times a variable. It is to be noted that the resistance as measured at the input terminals is a constant and equal to $R_{s}$.

\subsection{Division}

A method must next be found to divide a constant by a constant plus a variable. The circuit shown in figure 3 accomplishes this electrically. Again, subscripts have been chosen as a convenience to later work.

The equation for the voltage ratio is

$$
\frac{e_{0}}{e_{i}}=\frac{R_{s}}{R_{s}+R_{7}+X_{5} R_{5}}
$$

or

$$
\frac{e_{0}}{e_{i}}=\frac{R_{s} / R_{5}}{\left(R_{s}+R_{7}\right) / R_{5}+X_{5}}
$$

Equation (5) is of the proper form to solve a division problem of the type encountered in the solution of the refractive-index equation, that is, a constant divided by a constant plus a variable.

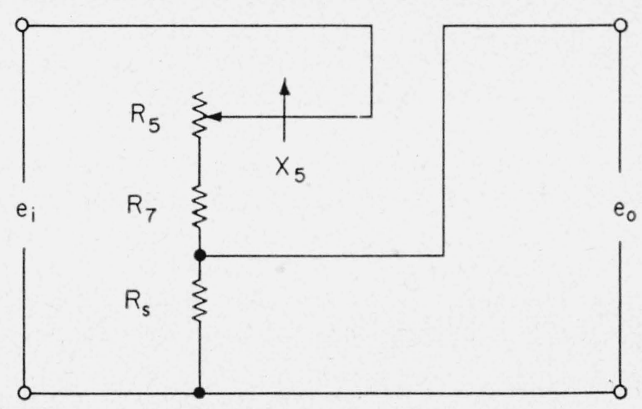

Figure 3. Division circuit. 


\section{A Circuit to Solve the Refractive-Index Equation}

\subsection{Limits on the Variables}

Before proceeding to combine the three basic circuits, it will be well to investigate each variable separately in the equation and discover the limits that may be placed on each of them. In addition, the variables in the equation must be modified so that they may be interpreted as the $X$ variables as used in the basic circuits. This requires that the maximum value of each variable be referred to a value of unity, and as such can be made equal to a percentage of rotation as expressed by the $X$ variables. Consideration will also be given toward estabishing limits on the variables such that standard dials graduated (or marked) with 10 major divisions to the revolution may be used.

The temperature, $T$, as found in the equation, represents the absolute temperature and may be written as $273+t$, where $t$ is in degrees centigrade. Because the data obtained from the recording instruments are in terms of degrees centigrade, it will be more convenient to have the dial marked in these same units. Investigation of the data currently available disclosed that temperatures to be encountered will lie between $\pm 50^{\circ} \mathrm{C}$. This range permits each revolution of a 10-turn potentiometer to represent a change of $10 \mathrm{deg}$, and the dial can be marked to read zero at its midscale position. With regard to the circuit, the temperature expression may be rewritten $T=100\left(2.23+X_{T}\right)$. As a check on the validity of this expression, consider the absolute temperature at $0^{\circ} \mathrm{C} . X_{T}$, or the fractional rotation, would equal 0.5 , and the expression would give an absolute temperature of $273^{\circ} \mathrm{K}$, as was to be expected.

Initial investigation of the pressure variable indicated that a range of 0 to 1,000 millibars would be adequate to accommodate all the current and future data. However, closer checks revealed that a range of 100 to 1,100 millibars would be more suitable. This range still permits assigning 100 millibars to each revolution of a 10-turn potentiometer, but requires that a constant be added to the circuit to take account of the 100-millibar shift. The expression for $p$ is written $p=1,000\left(0.1+X_{p}\right)$. Here again $X_{p}$ is the percentage of dial rotation, with the dial calibrated from 100 to 1,100 millibars. As an example, with the dial set for 600 millibars, $X_{p}$ would equal 0.5 , and $p$ would equal 600 .

The relative-humidity variable lends itself admirably to expression as a percentage rotation. Because it varies from 0 to 100 percent in the same manner as the $X$ variable, the dial may be graduated 0 to 100 and used directly. Investigation of the data disclosed that the complete range 0 to 100 would be required to accommodate all the data.

Although the $e_{s} / T$ variable will later be eliminated as an independent variable, its limits will be considered at this time. Using the 1951 Smithsonian
Meteorological Tables [5], $e_{s} / T$ was found to vary from 0.0003 at $-50^{\circ} \mathrm{C}$ to 0.3820 at $+50^{\circ} \mathrm{C}$. In order to use all the available rotation of a linear potentiometer, it will be necessary to express the variable as $0.3820 X e_{s} / T$ and use a specially marked dial to position the slider of the potentiometer.

\subsection{The Basic Circuits Combined}

With the above information in mind, eq (2) may be rewritten

$$
N=\frac{K_{1}}{100\left(2.23+X_{T}\right)}\left[1000\left(0.1+X_{p}\right)+0.3820 X_{\left(e_{s} / T\right)} X_{R H}\right]
$$

or

$N=\frac{10 K_{1}}{2.23+X_{T}}\left[0.1+X_{p}+0.3820 \frac{K_{2}}{1000} X_{\left(e_{s} / T\right)} X_{R H}\right]$.

The basic circuits will now be combined, and it will be shown that, by properly selecting the resistance values, the combined circuit can be used to solve the refractive-index equation in the form of eq (6).

Using the equations already established for the basic circuits, the equation for the combined circuit of figure 4 may now be written. The output voltage of the division circuit is applied as the input voltage to the addition circuit, and the variable $X_{3}$ is included in the last term of the equation for the addition circuit. Justification for this action is found by comparing the multiplication circuit of figure 1 with the right-hand portion of the addition circuit of figure 2.

The equation for the ratio of voltages in figure 4 is

$\frac{e_{0}}{e_{i}}=\frac{\frac{R_{4 \mathrm{a}}}{R_{5}}}{\frac{R_{s}+R_{7}}{R_{5}}+X_{5}}\left[\frac{R_{6 \mathrm{a}}}{R_{4 \mathrm{a}}}+X_{4}+\frac{R_{p} R_{2}}{R_{4 \mathrm{a}}\left(R_{1}+R_{2}\right)} X_{2} X_{3}\right]$.

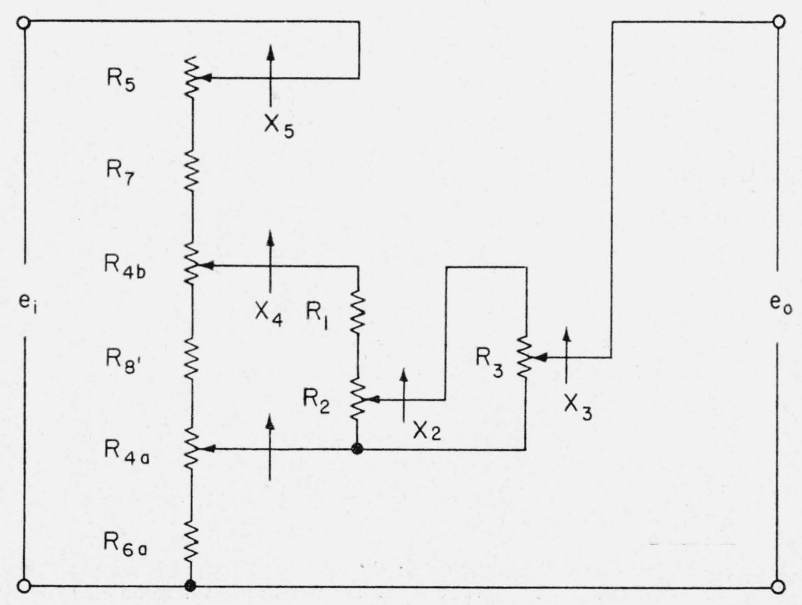

FIgure 4. Combined computation circuit. 
A comparison of eq (6) and (7) will show the similarity of the two equations. It is now only necessary to equate the resistance combinations in eq (7) to the constants in eq (6). The output to input voltage ratio is then equal to $N$.

\section{Elimination of $e_{\mathrm{s}} / T$ as an Independent Variable}

Up to this point $e_{s} / T$ has been considered as a separate variable in the equation. The curve plotted in figure $5,{ }^{4}$ shows that $e_{s} / T$ has explicit dependence on the temperature. This fact can be used to eliminate $e_{s} / T$ as an independent variable and consequently reduce the setup time for the computer.

As discussed in section 4.1., if a linear potentiometer is to be used to introduce the $e_{s} / T$ ratio, its dial must have a special marking. The same ratio may be established by using a linear dial and a potentiometer with an output function that matches the curve in figure 5. Unfortunately, the curve cannot be duplicated by any convenient mathematical expression. However, it is possible to approximate the curve by a series of straight lines connecting the circled points on figure 5. Although this approach does not yield a perfect solution at all points, the accuracy can be made as high as required by merely increasing the number of straight-line approximations.

A method known as resistance loading, or "padding", was used to alter the normally linear output of a 10-turn potentiometer. This linear relationship between resistance and shaft rotation can be altered

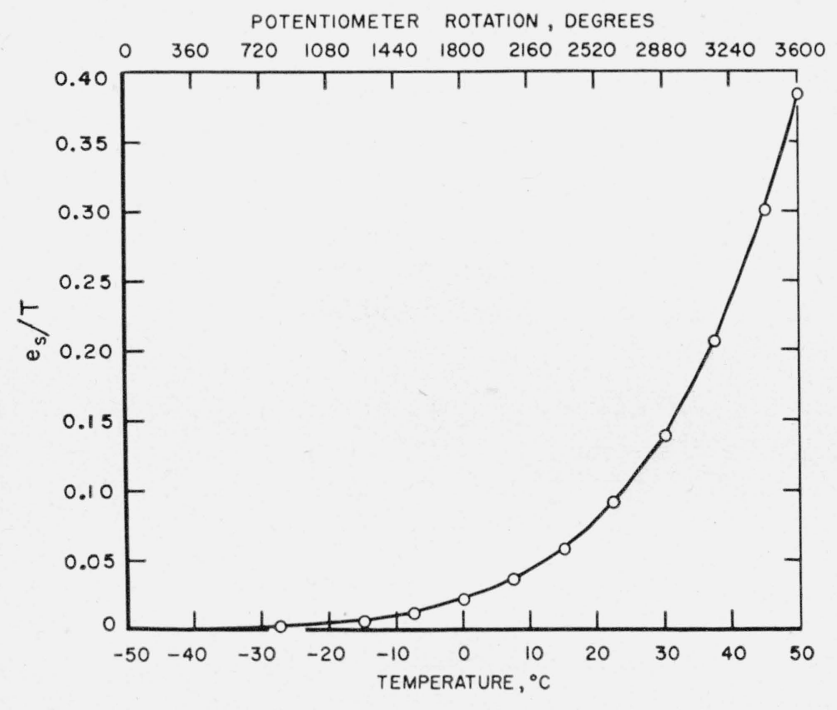

FiguRE 5. $e_{s} / T$ versus temperature.

Theoretical curve shown by the solid line, actual output function shown by the circles. $e_{s}$ is the saturation vapor pressure in millibars; $T$ is absolute temperature.

4 Data for this curve were taken from the 1951 Smithsonian Meteorological Tables [5]. by connecting padding resistors between tap points on the potentiometer coil so that the output will vary in a close approximation to the desired function. By properly choosing the resistance of the pads, the straight lines generated between the tap points can be made to cross the desired curve at two points. The number of tap points was chosen so that at no point would the series of straight lines deviate from the theoretical enough to contribute over $\frac{1}{4} \mathrm{~N}$ unit error to the final result.

Examination of the curve disclosed that taps located at the following degree positions would provide for the desired accuracy: $810,1260,1530$, $1800,2070,2340,2610,2880,3150$, and 3420 . A 2,000 -ohm potentiometer was selected for this modification, as 2,000 ohms was the lowest resistance, commercially available, 10-turn potentiometer that could be obtained for this purpose. Approximate resistance values were calculated to pad the 2,000 ohm potentiometer to the desired points on the curve, and it was found possible to hold the total resistance after padding to $200 \mathrm{ohms}$. The exact values of the padding resistances were determined by the voltage-comparison method described below.

A 500-ohm 10-turn potentiometer was placed in series with the tapped potentiometer $R_{2}$. A voltage was applied to the series combination, and the output voltage at the slider was read, potentiometer type precision voltmeter. The ratio of slider voltage was calculated for each tap point, and, starting from the lowest tap point, the values of the padding resistors were adjusted to make the voltage ratio check exactly with the desired value of $e_{s} / T$. The 500 -ohm potentiometer was adjusted after every change in padding resistance so as to keep the total series resistance constant and equal to $523.5 \mathrm{ohms}$. Because the maximum value of $e_{s} / T$ was to be 0.3820 , the $500-$ ohm potentiometer was replaced by a fixed resistance $\left(R_{1}\right)$ of $323.5 \mathrm{ohms}$ upon completion of the padding operation.

This method provided much greater accuracy than could have been obtained by calculating the values for the padding resistors. With the type of precision voltmeter used, it was possible to read the voltages to five-figure accuracy, and consequently the resistors could be changed to permit adjusting the voltage ratios at the tap points to an accuracy of 0.01 percent. Wire-wound resistors of the same temperature coefficient wire as that used in the potentiometer were specially constructed for this purpose.

Using the potentiometer just described, it is now possible to graduate its dial from $-50^{\circ} \mathrm{C}$ to $+50^{\circ} \mathrm{C}$ in exactly the same manner as the temperature dial. In fact, the dial can now be eliminated and the $e_{s} / T$ potentiometer can be connected directly to the shaft of the temperature potentiometer. This has in effect eliminated the $e_{s} / T$ variable, in that it now depends directly on the temperature dial for its setting. 


\section{Final Design Considerations}

\subsection{Choice of Solution Presentation}

As shown by eq (7), the computation circuit as developed to this point will provide a solution to the refractive-index equation in the form of a voltage ratio. A more convenient and accurate method of presenting the solution is to combine the composite circuit of figure 4 into a bridge circuit and use a sensitive null indicator to detect the bridge balance. Figure 6 shows the complete computer circuit diagram. Potentiometer $R_{9}$ and the fixed resistance $R_{10}$ constitute one arm of the bridge, and the combined computation circuit of figure 4 forms the other arm. $\quad R_{9}$ is the same type 10-turn linear potentiometer as used to introduce the variables. A linearly calibrated dial attached to $R_{9}$ was used to read $N$ directly. $R_{10}$ is a wire-wound resistor, as are all the other fixed resistances.

The null indicator used is of a special design having a maximum sensitivity of $1 / 4$-in. motion per microampere at about the zero position. Its response falls off logarithmically on either side of zero and thus provides automatic protection against excessive currents caused by large unbalances in the bridge circuit.

\subsection{Selection of Resistance Values}

Referring to figure 6 , the following expression for the voltage as measured at the slider of $R_{9}$ may be written:

$$
\frac{e_{0}}{e_{i}}=\frac{X_{9} R_{9}}{R_{9}+R_{10}} .
$$

With the circuit as shown at null balance, this expression may be equated to eq (7).

$$
X_{9}=\frac{\frac{R_{4 \mathrm{a}}\left(R_{9}+R_{10}\right)}{R_{5} R_{9}}}{\frac{R_{s}+R_{7}}{R_{5}}+X_{5}}\left[\frac{R_{6 \mathrm{a}}}{R_{4 \mathrm{a}}}+X_{4}+\frac{R_{p} R_{2}}{R_{4 \mathrm{a}}\left(R_{1}+R_{2}\right)} X_{2} X_{3}\right] .
$$

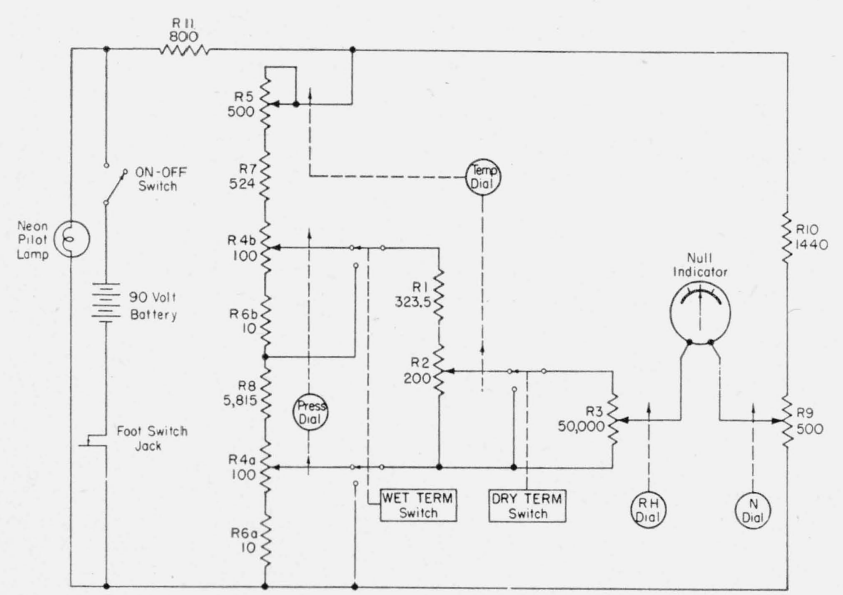

Figure 6. Computer-circuit diagram.
Both sides of this equation must be multiplied by 1,000 to permit solving for values of $N$ up to 1,000 . Investigation of the actual data disclosed that all the values of $N$ will be between 0 and 1,000. The dial attached to $R_{9}$ must therefore be graduated from 0 to 1,000 . Comparison of eq (6) and (9) requires that the following identities be satisfied in order to utilize the final circuit to solve the refractive-index equation:

$$
\begin{gathered}
1000 \frac{R_{4 \mathrm{a}}\left(R_{9}+R_{10}\right)}{R_{5} R_{9}}=10 k_{1}=776 . \\
\frac{\left(R_{\mathrm{s}}+R_{7}\right)}{R_{5}}=2.23 . \\
\frac{R_{6 \mathrm{a}}}{R_{4 \mathrm{a}}}=0.1 . \\
\frac{R_{p} R_{2}}{R_{4 \mathrm{a}}\left(R_{1}+R_{2}\right)}=\frac{K_{2}}{1000} 0.3820=1,8374 .
\end{gathered}
$$

also

$$
\begin{gathered}
R_{p}=\frac{\left(R_{1}+R_{2}\right)\left(R_{4 \mathrm{a}}+R_{8}^{\prime}\right)}{R_{1}+R_{2}+R_{4 \mathrm{a}}+R_{8}^{\prime}} . \\
R_{s}=R_{p}+R_{4 \mathrm{a}}+R_{6 \mathrm{a}} .
\end{gathered}
$$

There exist several combinations of the above resistances that will satisfy the above identities. The variable resistances or potentiometers must first be chosen on the basis of commercially available values. In general, they were chosen as small as possible in order to keep the over-all internal resistance of the circuit low with regard to the internal impedance of the null indicator. Low circuit resistance is necessary in this case to maintain sufficient sensitivity of the null indicator.

$R_{4 \mathrm{a}}$ and $R_{4 \mathrm{~b}}$ were chosen as 100 ohms each. This value, by eq (12), required that $R_{6 \mathrm{a}}=10 \mathrm{ohms}$, which was still of a size that could be conveniently wound. $R_{1}$ and $R_{2}$ have already been determined as 323.5 and $200 \mathrm{ohms}$, respectively. These values and the value for $R_{4 \mathrm{a}}$ when substituted in eq (13) give a value for $R_{p}$ of $481.00 \mathrm{ohms}$. Substituting this value in eq (14), along with the values for $R_{1}, R_{2}$, and $R_{4 a}$, gives a value of $5,825 \mathrm{ohms}$ for $R_{8}^{\prime}$. Solving eq (15) gives a value for $R_{s}$ of $591.00 \mathrm{ohms}$. Equation (11) was next investigated in order to determine a value for $R_{5}$. As $R_{7}$ cannot have a negative value, $R_{5}$ must be at least 278 ohms. The closest commercially available value of $500 \mathrm{ohms}$ was chosen for $R_{5}$. Using this value for $R_{5}$, eq (11) was solved, and $R_{7}$ was found to be $524.0 \mathrm{ohms}$.

Equation (10) was next used to determine the relative magnitude of $R_{9}$ and $R_{10}$. Solving this equation established the relationship

$$
\frac{R_{9}}{R_{9}+R_{10}}=0.25773
$$

$R_{9}+R_{10}$ was chosen to be approximately $2,000 \mathrm{ohms}$ so that excessive current would not be drawn from 
the voltage source and yet not be so great as to cause serious loss of sensitivity at the null indicator. This value for $R_{9}+R_{10}$ would indicate a value for $R_{9}$ of about 515 ohms. A resistance of 500 ohms was chosen for $R_{9}$ because resistors of that value are commercially available. This choice for $R_{9}$ required that $R_{10}$ equal 1,440 ohms. $R_{3}$ was selected as $50,000 \mathrm{ohms}$ in order to satisfy the previously established relationship that $R_{3}$ be at least 200 times $R_{2}$.

Figure 6 shows a resistance $R_{11}$ in series with the battery supply voltage. This resistor was used to reduce the bridge voltage to approximately 50 volts. At higher voltages a slight heating effect, along with corresponding resistance changes, was noted in some of the resistors.

\subsection{Wet- and Dry-Term Switches}

The refractive-index equation may be considered as being composed of two parts, a wet term equal to $K_{1} / T\left(K_{2} R H e_{s} / T\right)$ and a dry term equal to $\left(K_{1} / T\right) p$. Many of the applications that require the solution of the refractive-index equation also require the values for either the wet or dry contribution to $N$. Two switches were incorporated into the final circuit, which permit the computer to solve for either contribution and thereby greatly increase the usefulness of the computer.

In figure $6, R_{8}^{\prime}$ has been divided to form $R_{8}$ and $R_{6 b}$. $R_{6 b}$ has been made equal to $10 \mathrm{ohms}$ and $R_{8}$ equal to $5,815 \mathrm{ohms}$, thereby keeping the total resistance the same. With the switch connection between $R_{6 b}$ and $R_{8}$ closed, the pressure term is effectively reduced to zero, and the computer, after rebalancing, reads the wet term. In a similar manner, closing the dry-term switch, effectively makes the wet term zero, and the computer solves for the dry term only.

\section{Results and Discussion}

The computer herein described was built under the author's supervision at the National Bureau of Standards, Boulder, Colo., and is at present being actively used by their Radio Meteorology Group. The use of the computer has resulted in considerable savings in time over previously used methods.

\subsection{Accuracy}

A complete test was run on the computer, and the results of this test are given in table 1 . The table shows that the error is never greater than $\pm \frac{1}{2} N$ unit. This satisfies the original requirement. If greater accuracy were to be required, a greater number of tap points on the $e_{s} / T$ potentiometer would permit a closer approximation to the desired curve and consequently increase the final accuracy. The need for greater accuracy would also necessitate the use of dials with finer graduations.
Table 1. Final test data

\begin{tabular}{|c|c|c|c|c|}
\hline \multirow{2}{*}{$\begin{array}{c}\text { Tempera- } \\
\text { ture }\end{array}$} & \multirow{2}{*}{ Pressure } & \multirow{2}{*}{$\begin{array}{c}\text { Relative } \\
\text { humidity }\end{array}$} & \multicolumn{2}{|c|}{$N$} \\
\hline & & & Theoretical & Computer \\
\hline $\begin{array}{r}\circ C \\
-50 \\
-50 \\
+50 \\
+50 \\
+50 \\
+50 \\
0 \\
-14 \\
-12 \\
-10 \\
+40 \\
+42 \\
+44 \\
+46\end{array}$ & $\begin{array}{c}\text { Millibars } \\
100 \\
1,100 \\
100 \\
1,100 \\
100 \\
1,100 \\
600 \\
600 \\
600 \\
600 \\
600 \\
600 \\
600 \\
600\end{array}$ & $\begin{array}{r}0 \\
0 \\
0 \\
0 \\
100 \\
100 \\
50 \\
50 \\
50 \\
50 \\
50 \\
50 \\
50 \\
50\end{array}$ & $\begin{array}{r}34.80 \\
382.78 \\
24.02 \\
264.27 \\
465.51 \\
705.75 \\
185.86 \\
185.53 \\
185.11 \\
184.77 \\
289.29 \\
302.27 \\
315.96 \\
331.00\end{array}$ & $\begin{array}{r}34.6 \\
383.1 \\
24.0 \\
264.6 \\
465.7 \\
706.2 \\
185.8 \\
185.5 \\
185.2 \\
184.9 \\
289.7 \\
302.6 \\
315.9 \\
330.7\end{array}$ \\
\hline
\end{tabular}

\subsection{Speed}

The average operational time of the computer and operator was found to be approximately $10 \mathrm{sec}$, which is less than the original requirement of $12 \mathrm{sec}$. This time naturally varies with the individual operator. The shortest time of $8 \mathrm{sec}$ was recorded for an experienced operator and the longest time of 13 sec was recorded for a relatively inexperienced operator. This time could be decreased somewhat by using an automatic balance system to rotate the $N$ dial and thereby balance the bridge circuit more rapidly than can be done manually. This refinement was not deemed practical for the present computer due to the relatively longer time required to set in the variables.

\subsection{Convenience}

All of the original requirements on convenience have been incorporated in the final design. Figure 7 shows the computer. It is housed in a small sloping

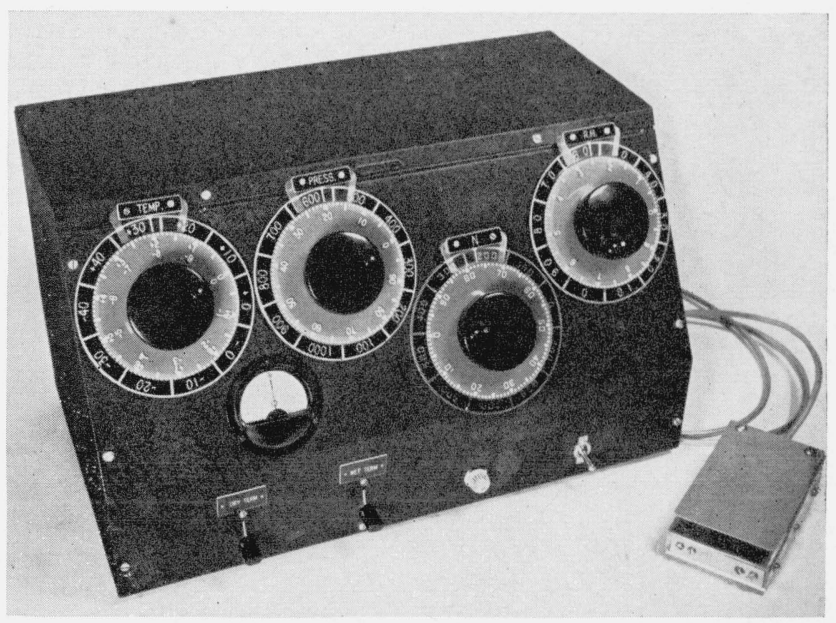

Figure 7. Refractive-index computer. 
front cabinet that can be placed on a desk or table and used in the same manner as a standard calculating machine. Because of heating effects, it was found desirable not to leave the battery connected to the bridge circuit continuously. With this in mind, a foot switch was incorporated in the final design so that the operator could energize the circuit as needed and still have both hands free to operate the dials and record data.

In figure 7 the various dials are set up to solve for $N$, with the temperature, $32.0^{\circ} \mathrm{C}$; pressure, 620 millibars; and RH, 62.5 percent. $N$ is read as 277.0.

\section{References}

[1] P. Debye, Polar molecules, sec. 3.4 (Chemical Catalog Co., New York, N. Y., 1929).

[2] E. K. Smith, Jr., and S. Weintraub, The constants in the equation for atmospheric refractive index at radio frequencies, J. Research NBS 50, 39 (January 1953) RP2385.

[3] D. M. Swingle, Nomograms for the computation of tropospheric refractive index, Proc. IRE [3] 41, 385 (March 1953).

[4] S. Weintraub, Slide rule for the computation of the radio refractive index of air, Electronics [1] 26, 182 (January 1953).

[5] Smithsonian meteorological tables, 6th revised ed., p. 351-352 (Smithsonian Inst., Washington, D. C., 1951).

Boulder, Colo., July 13, 1953. 